\title{
Tremor price dynamics in the world's network of stock exchanges
}

\author{
Jørgen Vitting Andersen ${ }^{1}$, Andrzej Nowak ${ }^{2}$, Giulia Rotundo ${ }^{3}$ and Lael Parrott ${ }^{4}$ \\ ${ }^{1}$ Institut Non Linéaire de Nice 1361 route des Lucioles, \\ Sophia Antipolis F06560 Valbonne, France \\ ${ }^{2}$ Department of Psychology, \\ Warsaw University, 00-183 Warsaw, Poland \\ ${ }^{3}$ Department of Economics, University of Tuscia, \\ via del Paradiso 47, 01100 Viterbo, Italy \\ and \\ ${ }^{4}$ Complex Systems Laboratory, Départment de géographie, Université de Montréal, \\ C.P. 6128 succursale "centre-ville” Montreal, Québec, Canada
}

(Dated: November 2, 2018)

\begin{abstract}
We use insight from a model of earth techtonic plate movement to obtain a new understanding of the build up and release of stress in the price dynamics of the world's stock exchanges. Nonlinearity enters the model due to a behavioral attribute of humans reacting disproportionately to big changes. This nonlinear response allows us to classify price movements of a given stock index as either being generated due to specific economic news for the country in question, or by the ensemble of the world's stock exchanges reacting together like a complex system. Similar in structure to the Capital Asset Pricing Model in Finance, the model predicts how an individual stock exchange should be priced in terms of the performance of the global market of exchanges, but with human behavioral characteristics included in the pricing. A number of the model's assumptions are validated against empirical data for 24 of the world's leading stock exchanges. We show how treshold effects can lead to synchronization in the global network of stock exchanges.
\end{abstract}

Like earthquakes, financial crises appear to be ever recurrent phenomena with the unfolding of a given crisis strongly dependent on the history that led up to the crunch. Whereas the continous build up of stress from techtonic plate movements is well understood to be at the origin of earthquakes, the causes behind financial distress remain unclear, with explanations often sought in singular events. Here we present a model that takes a holistic view of how the pricing takes place in the world's stock markets. In our model, each stock exchange is represented as a block in a network that links any two blocks with a spring of variable strength in a world wide network of stock exchanges. As will be explained below, the price movements of the stock exchanges are partially created by the stick-slip motion of the network of blocks, something very similar to ideas originally introduced by Burridge and Knopoff (BK) [1] to describe earthquakes caused by techtonic plate movement. This allows a direct study of memory effects in the global financial system, with stresses that build up over time and are released in sudden bursts much like what is seen during seismic activities of earthquakes. Thus, we emphasize a description where the price movements of any given stock market can not be solely understood by looking at the level of the individual stock exchange and propose that a proper characterisation needs to account for system-wide movements at the global level.

Our objective is to study how "stresses" in the global financial system of stock exchanges build up and propagate. In our model, stress enters the system because of price movements of the indicies represented by displacements of the blocks. Stress can either be locally generated due to economic news for a specific index, or globally generated due to the transfer of stress when a large movement happens for a given stock index. Similar to the BK model of earthquakes, we assume a "stick-slip" motion of the indicies so that only a large (eventually cumulative) movement of a given index has a direct impact in the pricing of the remaining indicies world wide. In this line of thinking "earthquakes" can happen in the global financial system because of cascades of big price movements originating from one corner of the globe and propagating world wide like falling bricks of dominos. We are thus representing the global financial system as a complex system, characterized by important memory effects and path dependence.

A key principle in finance states that as new information is revealed, it immediately becomes reflected in the price of an asset and thereby loses its relevance[2, 3]. We suggest to combine this principle with a behavioral trait 
which reflects the tendency of humans to reply in a nonlinear fashion to changes, placing emphasis on events with big changes and disregarding events with modest information content. This is in agreement with experiments made in psychology which have shown that humans react disproportionally to big changes, a phenomenon called change blindness since small changes go unnoticed[4, 5, 6].

As new information is produced at a given exchange, say the opening or closing price of that particular market, it becomes part of the information that other exchanges may or may not use in their pricing. With the existence of futures contracts, this information, as well as other economic news, is in principle priced in instantaneously, even outside the opening hours of exchanges. However if one uses the amount of trading volume as a proxy for the relevance of the reaction to new information, it is the opening (or respective closing) price that determines the most important moment where new information generated prior to the current exchange's trading session becomes priced in. Thus, in the following we will use the opening/closing (open/close) prices, which usually correspond to times when the trading volume is highest, as the values that become priced in.

Imagine a trader who at the opening of the Tokyo stock exchange tries to price in new world wide information coming from the other stock exchanges about what happened since the markets last closed in Tokyo. We conceive that she/he does so by taking into account both the release of local economic news in Japan (that happened since the previous day's close) as well as by seeking out news about how other markets performed after the markets closed in Tokyo. Because of the time zone differences, new information at the opening in Tokyo would include the price difference between the open and the close the day before for the European and American markets. For the Australian market, however, this would include the price difference between the close of the day before and the open the same day, since this market is the first market to open world wide, and opens before the Japanese markets. We postulate a universal behavioral mechanism in the pricing done by traders evaluating two different terms i) local economic news ii) big cumulative changes from other stock exchanges weighted by their importance (in terms of capitalization) and their relatedness (in terms of geographical positioning representing e.g., overlap of common economic affairs or importance as trading partners).

At time $t$, the trader of a given stock exchange $i$ estimates the price $P_{i}(t)$ of the index as $P_{i}(t)=P_{i}(t-$ 1) $\exp \left(R_{i}(t)\right)$, with $R_{i}(t)$ the return of stock exchange $i$ between time $t-1$ and $t$ :

$$
\begin{aligned}
R_{i}(t)= & \frac{1}{N_{i}^{*}} \sum_{j \neq i}^{N} \alpha_{i j} \Theta\left(R_{j}^{\text {cum }}(t-1)>R_{C}\right) R_{j}^{\text {cum }}(t-1) \beta_{i j}+\eta_{i}(t), \\
R_{j}^{\text {cum }}(t)= & \left(1-\Theta\left(R_{j}^{\text {cum }}(t-1)>R_{C}\right)\left[R_{j}^{\text {cum }}(t-1)+R_{j}(t)\right]\right. \\
& \left.N_{i}^{*}=\sum_{j \neq i}^{N} \Theta\left(R_{j}^{\text {cum }}(t-1)>R_{C}\right), \alpha_{i j}=1-\exp \left\{-K_{j} /\left(K_{i} \gamma\right)\right\}, \quad \beta_{i j}=\exp \left\{-\left(z_{i}-z_{j}\right) / \tau\right\} 3\right)
\end{aligned}
$$

$N$ is the total number of stock exchanges. The second term in (1), $\eta_{i}$, represents internal economic news only relevant for the specific index $i$, whereas the first term in (1) describes external news with large price movements of index $j$ having impact on index $i$. $t$ stands for the time of the close (respective open) of exchange $i$ whereas $t-1$ is the time of the last known information (close or open) of exchange $j$ known at time $t . \alpha_{i j}$ is a coefficient that describes the influence of stock index $j$ on stock index $i$ in terms of relative value of the capitalization $K_{i}, K_{j}$ of the two indicies. A large $\gamma(\gamma \gg 1)$ then corresponds to a network of the worlds indicies with dominance of the index with the largest capitalization $K_{\max }$. Presently this is the U.S. financial market, so choosing $\gamma$ large corresponds to the case where pricing in any country as external information only takes into account the movements of the U.S. markets. On the contrary a small $\gamma(\gamma \ll 1)$ corresponds to a network of indicies with equal strengths since $\alpha_{i j}$ becomes independent of $i, j$. In addition we assume that countries which are geographically close also have larger interdependence economically, as described by the coefficient $\beta_{i j}$ with $z_{i}-z_{j}$ the time zone difference of countries $i, j . \tau$ gives the scale over which this interdependence declines. Small $\tau(\tau \ll 1)$ then corresponds to a world where only indicies in the same time zone are relevant for the pricing, whereas large $\tau(\tau \ll 1)$ describes a global influence in the pricing independent of the difference in time zone. The structure of (1) is similar to the Capital Asset Pricing Model[7] since it predicts how an individual stock exchange should be priced in terms of the performance of the global market of exchanges, but with human behavioral characteristics included in the pricing.

$\Theta$ is a Heaviside function so only when the cumulative return of index $j, R_{j}^{c u m}$, exceeds a threshold return, $R_{C}$, does the index $j$ have a possible (depending on $\alpha_{i j}, \beta_{i j}$ ) impact on the pricing on index $i$. The factor $N_{i}^{*}$ in (1) means that the index $i$ takes into account an average impact among the indicies $j$ that have the condition in the Heaviside function fullfilled. (2) includes the key assumption in Finance that when new information arrives it 
becomes "priced in" in the price of an index. That is, after the information that $R_{j}^{c u m}>R_{C}$ has happened, and had an impact on index $i$, this information is deleted $\left(R_{j}^{c u m} \rightarrow 0\right)$. It should be noted however that memory effects are present in the model since it is the cumulative "stress" that determines when a block "slips". In Self Organized Critical (SOC) systems, memory is known to be an essential ingredient for the criticality of the system[8]. Formally (1)-(3) describes a 2D BK model of earth techtonic plate motion[9, 10]. It can be seen as an extension of the 2D Olami-Feder-Christensen (OFC) model[9] where each block is connected to all other blocks with $i, j$-dependent coupling constants $C_{i j}=\alpha_{i j} \beta_{i j}$. However, in the OFC model each block is only connected to its 4 neighbors and has only three ( $x, y, z$-dependent) coupling constants. In addition, in our model, "out of plane" stresses are randomly (in both sign and magnitude) introduced via $\eta_{i}$ at each block instead of the constant (same sign) pull of the OFC model. (112) gives therefore an interesting perspective of looking at the world's financial system as a complex system with self-organizing dynamics and possibly similar avalanche dynamics as can be observed for earthquakes.

The state of the network of the world's financial markets can according to (1-3) be characterised by the 4 variables $R_{C}, \gamma, \tau$ and the variance $\sigma$ of $\eta[11]$. It is possible to use maximum likelihood estimation to slave either $\gamma$ or $\tau$ to the remaining three parameters[12]. Slaving $\gamma$ to $R_{C}, \tau$ and $\sigma$ one finds:

$$
\begin{aligned}
\gamma & =\frac{\sum_{t=1}^{T} \sum_{i=1}^{N} 1 / N_{i}^{*}\left[R_{i}^{\text {data }}-\eta_{i}(t)\right] C_{i}(t-1)}{\sum_{t=1}^{T} \sum_{i=1}^{N}\left\{1 /\left(N_{i}^{*}\right)^{2} C_{i}(t-1)^{2}-\left[\eta_{i}(t)-R_{i}^{\text {data }}\right] C_{i}^{\prime}(t-1)\right\}} \\
C_{i}(t) & \equiv \sum_{i \neq j}^{N} K_{i} / K_{j} \Theta\left(R_{j}^{c u m}(t-1)>R_{C}\right) R_{j}^{\text {cum }}(t-1) e^{-\left(t_{i}-t_{j}\right) / \tau} \\
C_{i}^{\prime}(t) & \equiv \sum_{i \neq j}^{N}\left(K_{i} / K_{j}\right)^{2} \Theta\left(R_{j}^{\text {cum }}(t-1)>R_{C}\right) R_{j}^{\text {cum }}(t-1) e^{-\left(t_{i}-t_{j}\right) / \tau}
\end{aligned}
$$

To verify the hypothesis that large movements in the stock exchanges play a special role and tend to lead to clustering of large movements, we have used empirical data to calculate the conditional probability that a given stock market's daily return, $R=\log \left(p\left(t_{\text {close }}\right) / p\left(t_{\text {close }}-1\right)\right)$ has the same sign as the daily return of the world market of indicies[13]. From Fig. 1] it is clear that when the world wide index only exhibits small changes, little coherence is seen between the different country's movements. However, there appears to be a threshold after which large movements in the world wide index lead to sychronization of the individual country exchanges, with the majority tending to move in the same direction. Similar results have been found for individual stocks of a given stock market[14]. This reinforces our claim that the stock markets world wide should be considered as one system with large events playing a special role.

We then checked the specific assumptions in (1-3) that large movements of large capital indicies should have a particular impact on smaller capital indicies. Using the open-close return of the U.S. stock market gives a clear case to check for such a "large-move" impact. Since the Asian markets close before the opening of the U.S. markets, they should only be able to price in this information at their opening the following day. An eventual "large-move" U.S. open-close should therefore have a clear impact on the following close-open of the Asian markets. On the contrary, the European markets are still open when the U.S. market opens up in the morning, so the European markets have access to part of the history of the open-close of the U.S. markets. An eventual "large-move" U.S. open-close would therefore still be expected to have an impact on the following close-open of the European markets, but less so than for the Asian markets since part of the U.S. move would already be priced in when the European markets closed. Since the opening of the Asian markets by itself could influence the opening of the European markets, this futhermore could distort the impact coming from the U.S. markets. Figure $1 \mathrm{~b}$ illustrates again the crucial part of the assumption in our model that large moves are indeed special and have impact across markets. As expected, this effect is seen more clearly for the Asian markets compared to the European markets.

As an additional check on our assumption (1-3) we have constructed the difference $\eta_{i}=R_{i}(t)-$ $\frac{1}{N-1} \sum_{j \neq i}^{N} \alpha_{i j} \Theta\left(R_{j}^{c u m}(t-1)>R_{C}\right) R_{j}^{c u m}(t-1) \beta_{i j}$ from the empirical data of 24 of the world's leading stock exchanges using daily data since the year 2000. According to (1) this difference should be distributed according to a Gaussian distribution. We found the optimal parameters to be: $\left(\gamma=0.8, \tau=20.0, R_{C}=0.03, \sigma^{2}=0.0006\right)$. Fig 2 shows that for these parameter choices, our definition of price movements due to external (random) news does indeed fit a normal distribution. The obtained values of the optimal parameters suggest a fairly "global" network of stock exchanges with a large influence of pricing across time zones and pricing not only dominated by the largest capital index. A priori this seems in agreement with expectations. The value of $R_{c}$ is futhermore 
consistent with the estimate one can retrieve independently by visual expection of figure 1. Lastly, given these optimal parameters, we predicted the sign of the open/close for each stock exchange using the sign of $R_{i}^{\text {transfer }}$. Using in total 58244 events we found a very convincing $63 \%$ success rate of predicting the sign of the return of the open/close of a given stock exchange ex ante.

In analogy with earthquakes, $R_{i}^{\text {transfer }}$ can be thought of as describing seismic activity of stress propagating through the system. To see if such activity could be used to characterise special periods with high "tremor" activity of the world's stock exchanges we constructed $A(t) \equiv \sum_{i} R_{i}^{\text {transfer }}(t)$. As can be seen in figure 3 there is a striking tendency for large "tremor" activity during down periods of the market. That is, traders seem to worry more about large movements (positive as well as negative) of other countries in the "bear" market phase.

We have introduced a new model of pricing for the world's stock exchanges that uses ideas from finance[15] , physics and psychology. The model is an extended version of the Burridge-Knopoff model that originally was introduced to describe earth techtonic plate movement. Like Zumbach et al.[16] who suggested a "GuttenbergRichter" scale of financial market shocks, we have used the analogy with earthquakes to get a new understanding of the build up and release of stress in the world's network of stock exchanges. Nonlinearity entered the model due to a behavioral attribute of humans reacting disproportionately to big changes. As predicted, such a nonlinear response was observed in the impact of pricing from one contry to another. The nonlinear response allows a classification of price movements of a given stock index as either exogeneously generated due to specific economic news for the country in question, or endogeneously created by the ensemble of the world's stock exchanges reacting like a complex system. The approach could shed new light of dangers connected to systemic risks when large financial "shocks" propagate world wide[12] . 

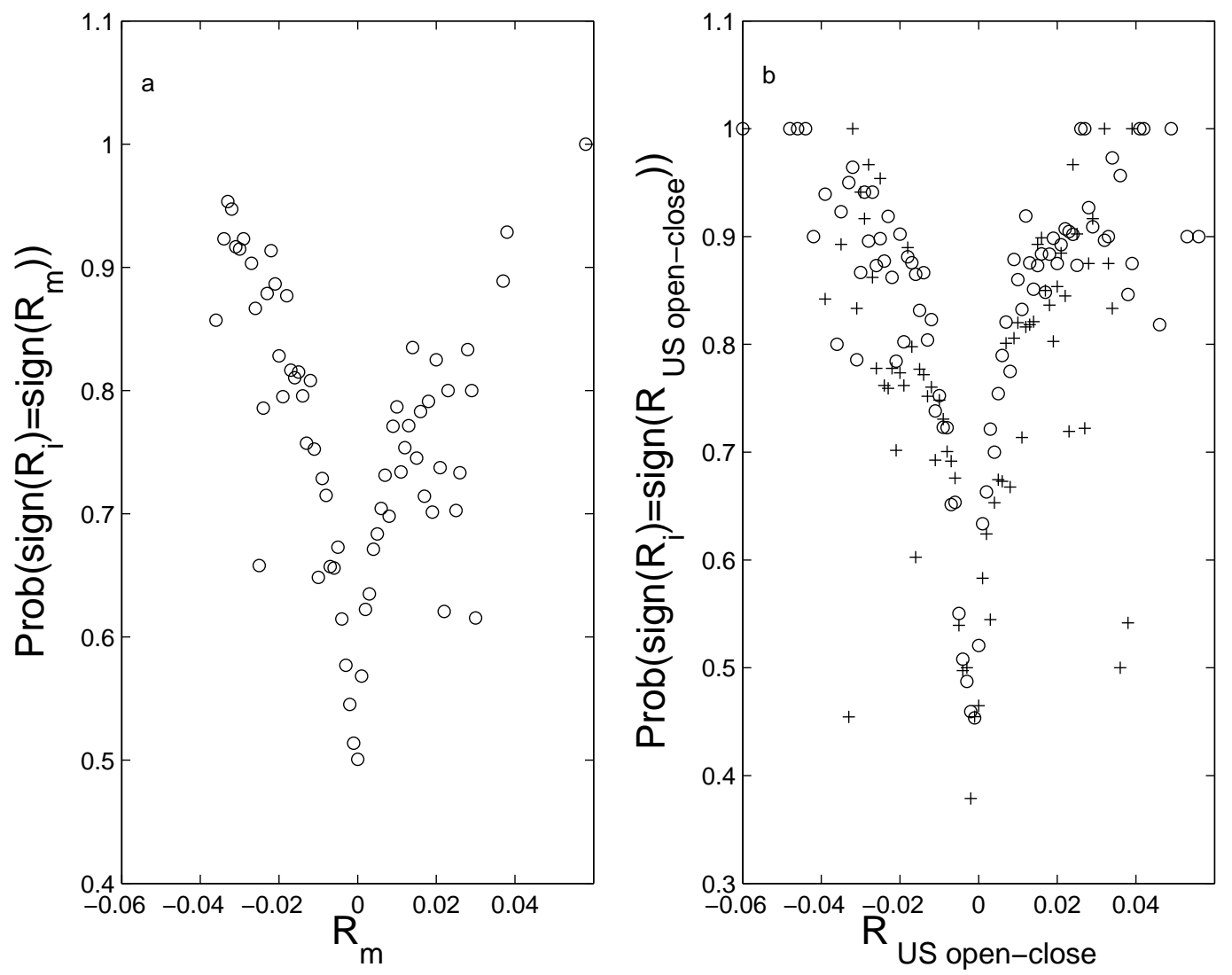

FIG. 1: a) Conditional probability that the daily return $R_{i}$ of a given country's stock market index has the same sign as the world market return defined by $R_{m} \equiv \sum_{j \neq i}^{N} \sum_{j \neq i}^{K_{j} R_{j}}$ with $K_{j}$ the capitalization of the $j$ 'th country's index. b) Conditional probability that the close-open (+: European markets; circles: Asian markets) return $R_{i}$ of a given country's stock market index following an U.S. open-close, has the same sign as the U.S. open-close return.

* Electronic address: vitting@ unice.fr

[1] R. Burridge and L. Knopoff, L. Bull. Sies. Soc. Amer. 57, 341, 1967.

[2] P. Samuelson, "Proof That Properly Anticipated Prices Fluctuate Randomly", Industrial Management Review 6, 41-49 (1965).

[3] E. Fama, "Efficient Capital Markets: A Review of Theory and Empirical Work”, Journal of Finance 25, 383-417 (1970).

[4] R. H. Jones, D. H. Crowell and L. E. Kapuniai, "Change Detection Model for Serially Correlated Data", Psychological Bulletin, Vol. 71, No. 5, 352-358 (1969).

[5] R. A. Rensink, "Change Detection", Annual Review of Psychology, Vol. 53, 245-277 February 2002.

[6] T. D. Lewin, N. Momen, S. B. Drifdahl and D. J. Simons, "Change Blindness, the metacognitive error of etimating change detection ability", Vision 7 (1,2,3), 397-413 (2000).

[7] J. L. Treynor "Toward a Theory of Market Value of Risky Assets ", Asset Pricing and Portfolio Performance: Models, Strategy and Performance Metrics. Risk Books, 15-22 (1999); J. Lintner "The Valuation of Risk Assets and the Selection of Risky Investments in Stock Portfolios and Capital Budgets ", Review of Economics and Statistics, 47 (1) 13-37 (1965); W. F. Sharpe "Capital Asset Prices: A Theory of Market Equilibrium under Condition of Risk", Journal of Finance, 19 (3) 425-442 (1964).

[8] P. Bak "How Nature Works: The Science of Self-Organized Criticality ", New York: Copernicus, ISBN 0-387-94791-4 (1996).

[9] Z. Olami, H.J.S. Feder and K. Christensen, "Self-Organized Criticality in a Continous, Nonconservative Cellular Automaton Modeling Earthquakes”,Phys. Rev. Lett. 68, 1244-1247 (1992).

[10] K.-T. Leung, J. Müller and J. V. Andersen, "Generalization of a Two-dimensional Burridge-Knopoff Model of Earthquakes 


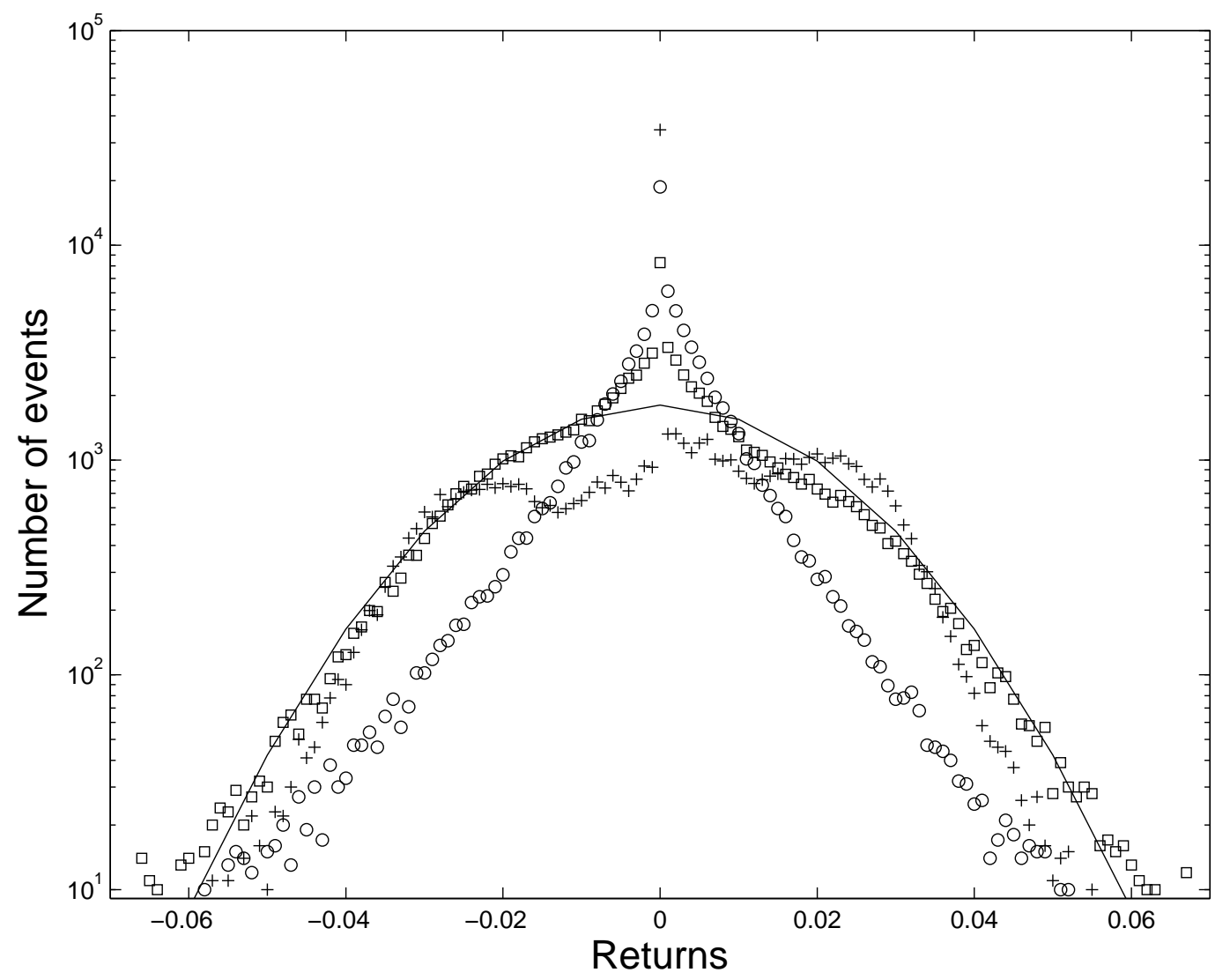

FIG. 2: Observed returns $R_{i}$ are shown by circles whereas the term $R_{i}^{\text {transfer }} \equiv \frac{1}{N-1} \sum_{j \neq i}^{N} \alpha_{i j} \Theta\left(R_{j}^{\text {cum }}(t-1)>\right.$ $\left.R_{C}\right) R_{j}^{\text {cum }}(t-1) \beta_{i j}$ are given by $+^{\prime} s$. Difference $\eta_{i} \equiv R_{i}^{\text {transfer }}-R_{i}$ (which according to (1)-(3) should be Gaussian distributed) is represented by squares. Solid line represents a normal distribution. The optimal parameters were found using the central part of the distribution of $\eta_{i}$ taking into account only events which had at least 10 occurencies.

", J. de Phys. I, 423, (1997).

[11] Assuming the same constant variance for all exchanges.

[12] J. Vitting Andersen, A. Nowak, G. Rotundo and L. Parrot, work in progress (2009).

[13] The data was downloaded from the website finance.yahoo.com and used the opening and closing price of the following 24 stock exchanges from 1/1/2000 to 1/10/2008: AORD (Australia), N225 (Japan), KS11 (South Korea) SSEC (China), HSI (Hong Kong), TWII (Taiwan), STI (Singapore), KLSE (Malysia), JKSE (Indonesia), BSESN (India), TA100 (Israel), CCSI (Egypt), FTSE (U.K.), FCHI (France), GDAX (Germany), SSMI ( Switzerland), MIBTEL (Italy), AEX (Netherlands), ATX (Austria), MERV (Argentine), BVSP (Brazil), GSPC (U.S.), GSPTSE (Canada) and MXX (Mexico).

[14] P. Cizeau, M. Potters and J.-P. Bouchaud, "Correlation structure of extreme stock returns", Quantitative Finance, Vol. 1, 217-222 (2001); F. Longin and B. Solnik, "Is the Correlation in international equity returns constant 1960-1990?" Journal of International Money and Finance, No.14m 3-26 (1995).

[15] J. Vitting Andersen, "Models of investment decision making in finance", "Encyclopedia of Complexity and System Science" Springer, in press 2009.

[16] G. Zumbach, M. Dacorogna, J. Olsen and R. Olsen "Measuring Shocks in Financial Markets", Int. J. Theo. App. Finance 3, 347-355 (2000); B. Maillet and T. Michel "An Index of Market Shocks Based on Multiscale Analysis ", Quantitative Finance 3, 88-97 (20030. 


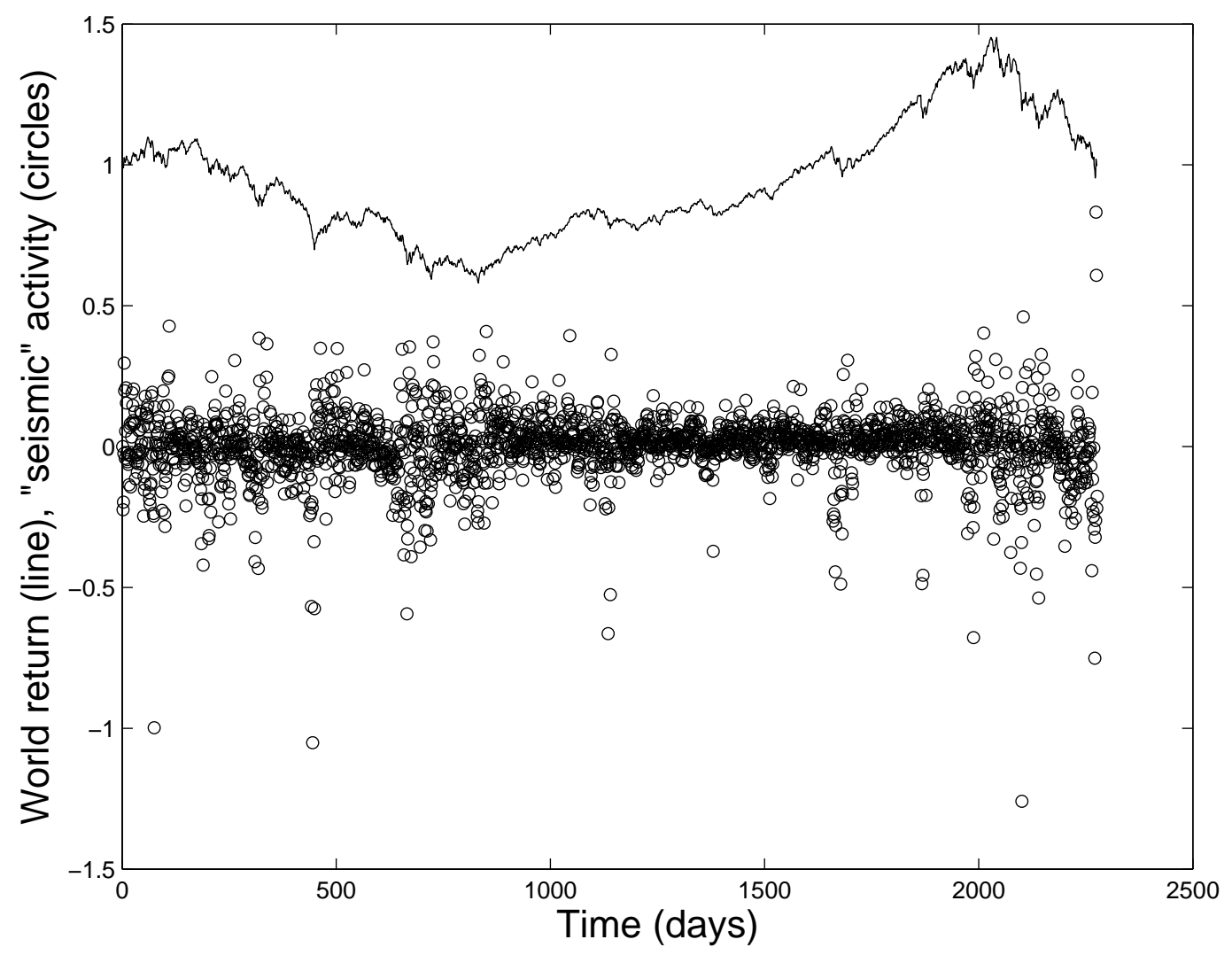

FIG. 3: Circles represent the term $A(t) \equiv \sum_{i} R_{i}^{\text {transfer }}(t)$ whereas the solid line is the world return index normalised according to capitalisation of the different stock indicies. 